\title{
Recognition of tumor antigens in 4T1 cells by natural IgM from three strains of mice with different susceptibilities to spontaneous breast cancer
}

\author{
MARIANA DÍAZ-ZARAGOZA, RICARDO HERNÁNDEZ-ÁVILA and PEDRO OSTOA-SALOMA \\ Departamento de Inmunología, Instituto de Investigaciones Biomédicas, \\ Universidad Nacional Autónoma de México, 04510 Ciudad de México, México
}

Received June 9, 2016; Accepted September 15, 2016

DOI: $10.3892 / \mathrm{ol} .2016 .5427$

\begin{abstract}
The issue of antibody responses to tumors is potentially important to cancer immunologists. Early detection of cancer represents one of the most promising approaches to reduce the growing cancer burden. Natural immunoglobulin (Ig)M antibodies have been associated with the recognition and elimination of cancerous and precancerous cells. Using natural IgM antibodies, the present study identified a set of antigens in healthy mice from three different strains and examined whether the global patterns of antibodies are able to discriminate between a condition of more or less susceptibility to breast cancer. The current study performed two-dimensional (2D) immunoblotting to detect antigens from 4T1 cells using natural IgM from serum of healthy female mice from three different strains. The $t$-test was used to analyze the total number of spots. There were no significant differences in the numbers of antigens recognized in each strain. However, differences in patterns were observed on 2D immunoblots among the three strains. The reactivity patterns of natural IgM antibodies to particular antigens exhibited non-random clustering, which discriminated between strains with different susceptibilities to spontaneous breast cancer. The results demonstrated that the patterns of reactivity to defined subsets of antigens are able to provide information regarding differential diagnosis associated with breast cancer sensitivity. Therefore, it may be concluded that it is possible to segregate the IgM humoral immune response toward cancer antigens according to the genetic background of individuals. In addition, it is possible to identify the recognized antigens that allow grouping or discriminate between the different
\end{abstract}

Correspondence to: Dr Pedro Ostoa-Saloma, Departamento de Inmunología, Instituto de Investigaciones Biomédicas, Universidad Nacional Autónoma de México, Apartado Postal 70228, 04510 Ciudad de México, México

E-mail:postoa@unam.mx

Key words: breast cancer, immunoglobulin M, 4T1 cells, two-dimensional immunoblot, mice strains
IgM antibodies expressed. The possible association between a particular antigen and cancer susceptibility requires further study, but the methodology exposed in the present study may identify potential candidates for this possible association.

\section{Introduction}

A growing body of evidence suggests that patients with cancer develop antibodies against a variety of tumor-associated antigens (TAA) (1-9). This has led to the theory that autoantibodies may be used as a tool for the early diagnosis of cancer. Natural immunoglobulin (Ig)M antibodies have been associated with the recognition and elimination of cancerous and precancerous cells $(10,11)$. The majority of research has focused on establishing cancer biomarkers using IgG for the diagnosis or treatment of breast cancer, while IgM autoantibodies have been insufficiently studied despite their relevance in the early recognition of tumor antigens $(12,13)$. Strains of mice have been demonstrated to have different susceptibilities to spontaneous breast cancer, $\mathrm{DBA} / 2 \mathrm{~J}$ being one of the most susceptible, C57BL/6J one of the most resistant and BALB/c being moderately susceptible (14).

The aim of the present study was to analyze the patterns of recognition of 4T1 cell antigens using natural $\operatorname{IgM}$ from the sera of mice with different levels of susceptibility to spontaneous cancer, and to determine if there is any difference in tumor recognition patterns among the strains in order to deduce the putative natural IgM-recognizable antigens characteristic of the different levels of cancer susceptibility.

\section{Materials and methods}

Cell extracts. 4T1 mouse tumor cells $\left[\right.$ ATCC $^{\circledR}$ CRL-2539; donated by Dr Karen Manucharyan from the Instituto de Investigaciones Biomédicas, Universidad Nacional Autónoma de México (UNAM), Ciudad de México, Mexico] was cultured in RPMI 1640 medium supplemented with $10 \%$ fetal bovine serum (Gibco; Thermo Fisher Scientific, Inc., Waltham, MA, USA) and $1 \%$ streptomycin/penicillin/amphotericin mixture (Gibco; Thermo Fisher Scientific, Inc.) in $25 \mathrm{~cm}^{2}$ culture dishes (Corning Incorporated, Corning, NY, USA), and incubated at $37^{\circ} \mathrm{C}$ in an atmosphere of $98 \%$ humidity and $5 \% \mathrm{CO}_{2}$. 
Cultures at a confluence of 70-90\% were collected by scraping and protein extracts were obtained for two-dimensional (2D) electrophoresis as previously described $(12,13)$.

Mice and serum samples. A total of 10 healthy females (8 weeks old) of each BALB/c, C57BL/6, and DBA/2J mouse strains were kept in the animal facilities at the Instituto de Investigaciones Biomédicas, UNAM, under controlled conditions of temperature $\left(22^{\circ} \mathrm{C}\right)$, a relative humidity of $50-60 \%$ and $12 \mathrm{~h}$ dark-light cycles, with lights on between 7:00 a.m. and 9.00 p.m. The mice had free access to food and water ad libitum. The Ethics Committee of the Institute of Biomedical Research, UNAM approved this protocol (permission no. 2015-175). The mice were tail-bled on one occasion. The blood was incubated at $4{ }^{\circ} \mathrm{C}$ for $30 \mathrm{~min}$ and centrifuged at $1,306 \mathrm{x} g$ for $10 \mathrm{~min}$ to obtain the serum, which was stored at $-80^{\circ} \mathrm{C}$ until use.

Immunoblot analysis of 2D images. 2D immunoblots and image analysis were performed as previously described $(12,13)$. Briefly, the 2D immunoblots were digitalized on a HP Scanjet G4050 scanner with a resolution of $300 \mathrm{dpi}$ in a TIFF file format. All 2D immunoblots were analyzed using the same settings for brightness, contrast and color to minimize bias. The images were transferred to Adobe Photoshop CS5 (Adobe Systems Europe, Ltd., Maidenhead, UK) to match them according to spots present on all 2D immunoblots. The TIFF images were converted to .1sc format, as required for analysis in PDQuest ${ }^{\mathrm{TM}}$ 2-D Analysis Software (Bio-Rad Laboratories, Inc., Hercules, CA, USA). Master images were created from the duplicates of the 2D immunoblot images. Numbers of spots and their coordinates were determined on the 2D immunoblots.

Clustering. To analyze the patterns of the IgM antibodies, a clustering algorithm was applied. The 2D immunoblot images were analyzed as previously described (13). Each 2D immunoblot master was divided into 10 columns (pHi) x10 rows (molecular weight, $\mathrm{kDa}$ ). In each grid, matrices were established, assigning a score of 0 if there was no spot in the cell and 1 if there were $\geq 1$ spots. The matrix was converted into a vector by placing the $n$-th row immediately after its predecessor. Thus, instead of a 10x10 matrix, a vector was generated with 100 places containing values of 0 and 1 . This vector was used as the input for a python script to perform complete linkage clustering with the hcluster package 0.2.0 (15). For this analysis, the city-block metric was chosen, in which the distance between two points is the sum of the absolute differences of their Cartesian coordinates. The resulting hierarchical clustering was presented as a dendrogram.

Statistical analysis. A paired two sample $t$-test for means was used to analyze the total number of spots, and was performed using Microsoft Office Excel 2010 (Microsoft Corporation, Redmond, WA, USA). $\mathrm{P}<0.05$ was considered to indicate a statistically significant difference.

\section{Results and Discussion}

When tested on the 4T1 cell antigens, the sera from the mice in all three groups displayed extremely different $\operatorname{IgM}$ reactivity patterns. The master images obtained from the immunoblots subsequent to processing with the PDQuest program exhibited large and notable disparities in antigen recognition among the three strains (Fig. 1). According to the average number of spots, no significant differences in recognition spot numbers were observed among the C57BL/6J and DBA/2J strains $(\mathrm{P}=0.9567)$. However, significant differences in recognition spot numbers were observed among the $\mathrm{BALB} / \mathrm{c}$ and DBA/2J strains $(\mathrm{P}=0.017)$ and the $\mathrm{BALB} / \mathrm{c}$ and C57BL/6J strains ( $\mathrm{P}=0.0053$ ) (Fig. 2). This indicates that the number of spots, reflecting the number of active clones of IgM, is not decisive to determine susceptibility. In addition, when the spots were analyzed according to their positions on the blots, interspecies variations were much more evident than individual intraspecies disparities.

The 2D immunoblots presented in Fig. 1 demonstrate notable differences in the patterns of spot recognition between the DBA/2J and C57BL/6J strains. The C57BL6 serum was markedly different from the DBA and BALB/c sera, confirming the presence of strain-specific natural $\operatorname{IgM}$ antibody repertoires. If the reactivity of the natural IgM antibodies were merely neutral, it may be expected that the different strain serum should exhibit a similar degree and scope of IgM acuteness. When the 2D immunoblots were converted to digital signatures and processed in order to group them according to clustering algorithm (13), the resulting dendrogram grouped the individuals almost perfectly according to their respective strains (Fig. 3). This demonstrates that the method perceived natural IgM antibody repertoire differences. With one exception, all animals clustered according to their strain genotype. All of them constituted homogeneous groups according to their immunoreactivity. It appears that, rather than representing random noise in the system, natural IgM antibodies are a repertoire selected predominantly by the expression of a developmental genetic program for $\mathrm{V}$ gene expression, without ligand-dependent selection of clonal reactivities, which, according to Nobrega et al (16), is designated as the 'immunological homunculus'.

Notably, natural IgM antibody reactivities were selectively directed towards a defined subset of all 4T1 antigens, regardless of the amount of antigenic protein. This demonstrates that the binding of natural IgM may be specific.

These results agree with those reported in (16), whereby different IgM immunoreactivities of the BALB/c, DBA/2J and $\mathrm{C} 57 \mathrm{BL} / 6 \mathrm{~J}$ strains towards mouse liver extract antigens were observed. The results of the current study reinforce the notion of the genetic control of, in this case, the natural IgM antibody repertoire. It appears that at birth there is a finite number of particular sets of individually manifested IgMs that may be grouped according to species-specific criteria; the natural IgM from mice of different strains react differently to the presentation of an extract of cancer cells (tumor antigens) to which they had not been previously exposed. As DBA/2J, C57BL/6J and BALB/c mice have naturally different susceptibilities to breast cancer (14), it may be assumed that IgM is able to distinguish between antigens contributing in greater or lesser measure to susceptibility to cancer.

The methodology used in the present study allowed identification (by molecular weight and isoelectric point) of the antigens that were more frequent, possibility indicating 


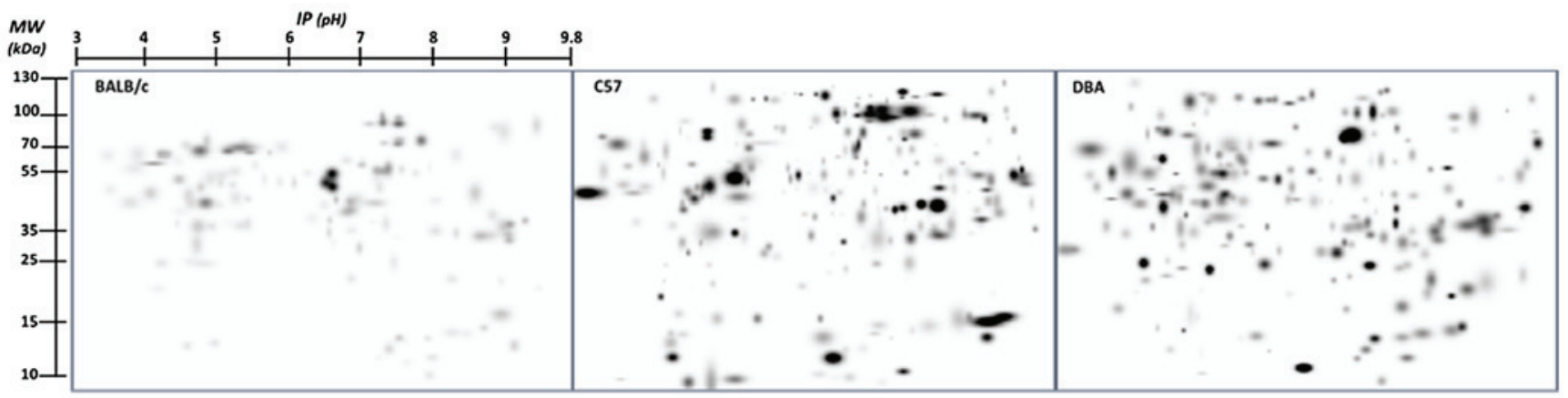

Figure 1. Master images of immunoglobulin M two-dimensional immunoblots from female mice of DBA/2J, C57BL/6J and BALB/c strains. MW, molecular weight; IP, isoelectric point.

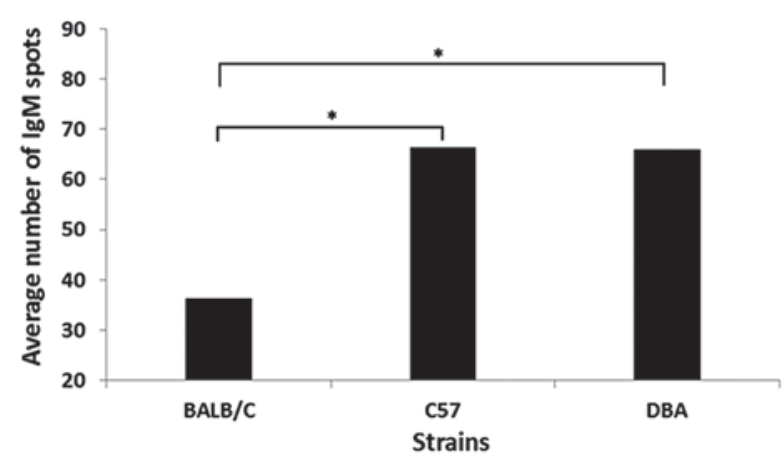

Figure 2. Average number of spots on two-dimensional immunoblots recognized by natural IgM in sera from the DBA/2J, C57BL/6J and BALB/c female mice. No significant differences in recognition spot numbers were observed among the $\mathrm{C} 57 \mathrm{BL} / 6 \mathrm{~J}$ and $\mathrm{DBA} / 2 \mathrm{~J}$ strains $(\mathrm{P}=0.9567)$. However, significant differences in recognition spot numbers were observed among the $\mathrm{BALB} / \mathrm{c}$ and $\mathrm{DBA} / 2 \mathrm{~J}$ strains $(\mathrm{P}=0.017)$ and the $\mathrm{BALB} / \mathrm{c}$ and $\mathrm{C} 57 \mathrm{BL} / 6 \mathrm{~J}$ strains ( $\mathrm{P}=0.0053)$. IgM, immunoglobulin $\mathrm{M}$. ${ }^{*} \mathrm{P}<0.05$.

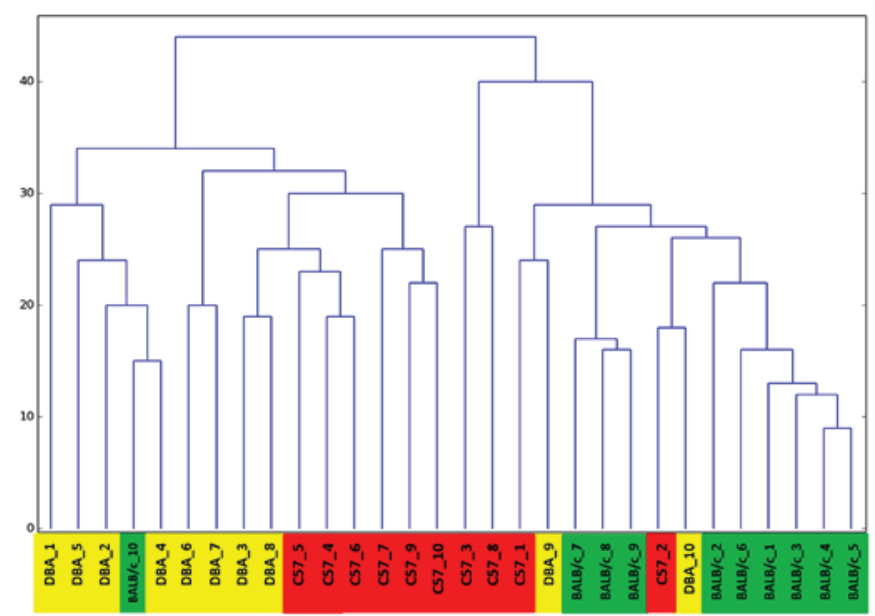

Figure 3. Dendogram obtained upon processing the immunological signatures of individual female mice of the DBA/2J, C57BL/6J and BALB/c strains. The individuals were grouped according to strain susceptibility to spontaneous cancer.

that they are fundamental in the differences among the strain patterns (Fig. 4; Table I). The identification of the susceptibility-specific antigens is underway in future studies. The determination of patterns of susceptibility to breast cancer in raised mice is only a first step in defining the susceptibility
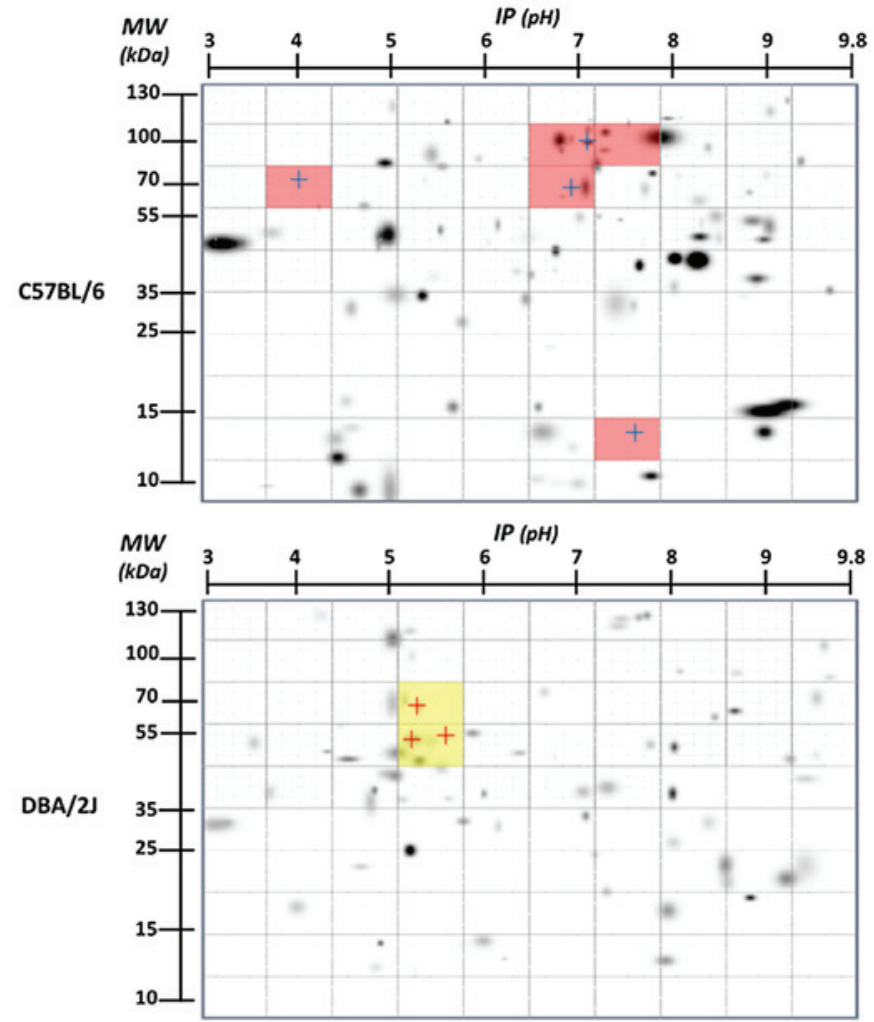

Figure 4. Images showing the exclusive immunoglobulin $\mathrm{M}$ spots in the most susceptible (DBA/2J) and less susceptible (C57BL/6) strains. Images were obtained subsequent to removal of shared spots. Crosses indicate the most frequent spots in each strain. MW, molecular weight; IP, isoelectric point.

Table I. Frequency, isoelectric point and molecular weight of the antigens marked in Fig. 4.

\begin{tabular}{ccccc}
\hline Strain & Spot N & Frequency, \% & $\begin{array}{c}\sim \text { Isoelectric } \\
\text { point }\end{array}$ & $\begin{array}{r}\sim \text { Molecular } \\
\text { weight, } \mathrm{kDa}\end{array}$ \\
\hline C57 & 1 & 90 & 4.05 & 73 \\
& 2 & 100 & 7.1 & 103 \\
& 3 & 100 & 6.9 & 69 \\
& 4 & 90 & 7.8 & 13.5 \\
DAB & 1 & 90 & 5.2 & 52 \\
& 2 & 80 & 5.3 & 68 \\
& 3 & 80 & 5.6 & 53 \\
\hline
\end{tabular}


that humans present to cancer, and the question remains whether patterns of natural IgM antibodies in humans may be used to anticipate susceptibility to breast cancer. The possible functions of natural IgM antibodies with generally greater or lesser resistance in breast cancer require further investigation, but at present their potential for immunodiagnosis is clear.

In conclusion, the results of the current study demonstrate that it is possible to segregate the $\operatorname{IgM}$ humoral immune response toward cancer antigens according to the genetic background of individuals. In addition, it is possible to identify the recognized antigens that allow grouping or discriminate between the different IgM antibodies expressed. The possible association of a particular antigen with the susceptibility to cancer requires further study, but the methodology applied in the present study may allow the unveiling of candidates for this possible association.

\section{Acknowledgements}

The current study received financial support from the Consejo Nacional de Ciencia y Tecnología Mexico, Ciudad de México, México (grant no. 151747) and the Programa de Apoyo a Proyectos de Investigación e Innovación Tecnológica (PAPITT), Dirección General de Asuntos del Personal Académico (DGAPA), Universidad Nacional Autónoma de Mexico, Ciudad de México, México (grant no. IN201715).

\section{References}

1. Tabernero MD, Lv LL and Anderson KS: Autoantibody profiles as biomarkers of breast cancer. Cancer Biomark 6: 247-256, 2010

2. Tan HT, Low J, Lim SG and Chung MC: Serum autoantibodies as biomarkers for early cancer detection. FEBS J 276: 6880-6904, 2009.

3. Desmetz C, Bascoul-Mollevi C, Rochaix P, Lamy PJ, Kramar A, Rouanet P, Maudelonde T, Mangé A and Solassol J: Identification of a new panel of serum autoantibodies associated with the presence of in situ carcinoma of the breast in younger women. Clin Cancer Res 15: 4733-4741, 2009.
4. Anderson KS, Ramachandran N, Wong J, Raphael JV, Hainsworth E, Demirkan G, Cramer D, Aronzon D, Hodi FS, Harris L, et al: Application of protein microarrays for multiplexed detection of antibodies to tumor antigens in breast cancer. J Proteome Res 7: 1490-1499, 2008.

5. Tan EM and Zhang J: Autoantibodies to tumor-associated antigens: Reporters from the immune system. Immunol Rev 222: 328-340, 2008

6. Lu H, Goodell V and Disis ML: Humoral immunity directed against tumor-associated-antigens as potential biomarkers for the early diagnosis of cancer. J Proteome Res 7: 1388-1394, 2008.

7. Finn OJ: Immune response as a biomarker for cancer detection and a lot more. N Engl J Med 353: 1288-1290, 2005.

8. Storr SJ, Chakrabarti J, Barnes A, Murray A, Chapman CJ and Robertson JF: Use of autoantibodies in breast cancer screening and diagnosis. Expert Rev Anticancer Ther 6: 1215-1223, 2006.

9. Anderson KS and LaBaer J: The sentinel within: Exploiting the immune system for cancer biomarkers. J Proteome Res 4: 1123-1133, 2005

10. Vollmers HP and Brändlein S: Natural antibodies and cancer. N Biotechnol 25: 294-298, 2009.

11. Díaz-Zaragoza M, Hernández-Ávila R, Viedma-Rodríguez R, Arenas-Aranda D and Ostoa-Saloma P: Natural and adaptive IgM antibodies in the recognition of tumor-associated antigens of breast cancer (Review). Oncol Rep 34: 1106-1114, 2015.

12. Díaz-Zaragoza M, Hernández R and Ostoa-Saloma P: 2D immunoblots show differential response of mouse IgG and IgM antibodies to antigens of mammary carcinoma $4 \mathrm{~T} 1$ cells. Cancer Cell Int 14: 9, 2014.

13. Díaz-Zaragoza M, Hernández-Ávila R, Govezensky T, Mendoza L, Meneses-Ruíz DM and Ostoa-Saloma P: Comparison patterns of $4 \mathrm{~T} 1$ antigens recognized by humoral immune response mediated by IgG and IgM antibodies in female and male mice with breast cancer using 2D-immnunoblots. Immunobiology 220: 1050-1058, 2015.

14. Hoag WG: Spontaneous cancer in mice. Ann N Y Acad Sci 108: 805-831, 1963.

15. Eads D: hcluster 0.2.0: A hierarcial clustering package for Scipy. https://pypi.python.org/pypi/hcluster/0.2.0. Accessed March 4, 2016.

16. Nobrega A, Haury M, Grandien A, Malanchère E, Sundblad A and Coutinho A: Global analysis of antibody repertoires. II. Evidence for specificity, self-selection and the immunological 'homunculus' of antibodies in normal serum. Eur J Immunol 23: 2851-2859, 1993 